\title{
Presence of focal and multifocal paratuberculosis lesions in mesenteric lymph nodes and the ileocaecal valve of cattle positive to the tuberculin skin test
}

\author{
A. Balseiro ${ }^{\text {a }}$, J.M. Prieto ${ }^{\mathrm{a}, *}$, A. Espí ${ }^{\mathrm{b}}$, V. Perez ${ }^{\mathrm{b}}$, J.F. García Marín ${ }^{\mathrm{b}}$ \\ ${ }^{a}$ Servicio Regional de Investigatión y Desarrollo Agroalimentario (SERIDA), Laboratorio de Sanidad Animal, Jove, Gijón, Asturias 33299, Spain \\ ${ }^{\mathrm{b}}$ Medicina Animal (Anatomía Patológica), Facultad de Veterinaria de León. Campus de Vegazana, s/n, 24075 León, Spain
}

Accepted 14 November 2002

Keywords: Cattle; Mycobacterium avium paratuberculosis; Immunohistochemical; Tuberculin skin test

The tuberculin skin test is commonly used in tuberculosis (TB) eradication programmes in cattle (Monaghan et al., 1994). The detection of false-positive reactors is important particularly in areas where there is a relatively low incidence of the disease, and can be attributed, at least in part, to immunological cross-reactivity with other mycobacteria infections. Of particular concern is Mycobacterium avium subspecies paratuberculosis (Map) infection, which is characterized by a chronic granulomatous enteritis in ruminants. The prevalence of Map seems to be increasing in Spain where it now accounts for approximately $30.4 \%$ of positive reactors in cattle (Juste et al., 2000). Paratuberculosis immunization is also know to interfere with the diagnosis of bovine TB when single skin test is performed (Kohler et al., 2001). In order to differentiate between both mycobacterial diseases, a comparative skin test has to be carried out and the response to avian purified protein derivative (PPD) is greater than to bovine PPD in paratuberculosis infected cattle (Chiodini et al., 1984). The aim of the present study was to describe the lesions associated with paratuberculosis in adult cattle positive to the single tuberculin skin test, and to compare these with cattle negative to the tuberculin test.

During the 11-month period of study, from February to December 1999, a total of 134 cattle, 4-9 years of age, was examined. Ninety-one (group 1) were positive tuberculin reactors to the single skin test, selected from 49 herds. Tuberculin skin test was performed by the in-

\footnotetext{
${ }^{*}$ Corresponding author. Fax: +34-985327811.

E-mail address: jmprieto@serida.org (J.M. Prieto).
}

tradermal inoculation of $0.1 \mathrm{~mL}$ of bovine PPD (CZ Veterinaria) in the cervical area and the skin thickness was measured at 0 and $72 \mathrm{~h}$ post-inoculation. Using standard interpretation, we classified cattle as positive reactors if there was an increase in skin thickness of $5 \mathrm{~mm}$ or more. The remaining 43 animals (group 2), which were negative to the tuberculin test, were randomly selected throughout the year from 39 herds. None of the cattle selected had any clinical signs of paratuberculosis (e.g., diarrhoea, decreased milk production, emaciation, or anorexia). No macroscopic or microbiologic evidence of infection with $M$. bovis was found in any of the cattle examined.

Samples from the ileocaecal valve and mesenteric lymph nodes were fixed in $10 \%$ neutral formalin and embedded in paraffin using standard procedures. Serial sections $5 \mu \mathrm{m}$ thick were cut and stained with haematoxylin and eosin and Ziehl-Neelsen methods (ZN). Stained sections were examined for the presence of typical paratuberculous lesions and acid-fast bacilli. For the immunohistochemical study, $5 \mu \mathrm{m}$ sections were cut and immunostained using the peroxidase-antiperoxidase (PAP) method. The sections were incubated with specific rabbit-antiserum at a dilution of 1:1000, prepared previously by the hyperimmunization of two rabbits with a sonic extract of a local Map strain (A-82) isolated from the intestinal tissues of a cow. Specificity was considered taking into account the histological features of the lesions, the location and the presence of immunohistochemical positivity.

Paratuberculosis lesions were classified, as proposed by Pérez et al. (1996) in sheep, on the basis of the 
presence, intensity and distribution (focal, multifocal or diffuse) of granulomatous lesions. The granuloma were formed by 5-20 macrophages, sometimes mixed with small number of lymphocytes and occasionally a few giant cells, with no more than four nuclei. Of the 134 cattle examined, $26(28.5 \%)$ from group 1 and 12 $(27.9 \%)$ from group 2 showed lesions associated with Map infection although no significant differences were found between the two groups (Table 1). Granulomas were located exclusively in the interfollicular areas of the lymphoid tissue (focal lesion) in $15(16.4 \%)$ from group 1 and $7(16.2 \%)$ from group 2. Other granulomas were located in the adjacent lamina propria of the ileocaecal valve (multifocal lesion) in $11(12.0 \%)$ from group 1 and $5(11.6 \%)$ from group 2 (Table 2$)$. The number of

Table 1

Presence of histological lesions associated with M. a. paratuberculosis infection in animals and herds, according to their response to tuberculin skin test (group 1, positive; group 2, negative)

\begin{tabular}{llllll}
\hline \multirow{2}{*}{ Group } & Animals & & & \multicolumn{2}{l}{ Herds } \\
\cline { 2 - 3 } \cline { 5 - 6 } & $\begin{array}{l}\text { Number } \\
\text { (total) }\end{array}$ & $\%$ & & $\begin{array}{l}\text { Number } \\
\text { (total) }\end{array}$ & $\%$ \\
\hline 1 & $26(91)$ & 28.5 & & $17(49)$ & 34.6 \\
2 & $12(43)$ & 27.9 & & $12(39)$ & 30.7 \\
\hline
\end{tabular}

Table 2

Number and percentage of cattle showing paratuberculosis lesions distributed according to lesion categories and their response to tuberculin skin test (group 1, positive; group 2, negative)

\begin{tabular}{lll}
\hline Lesion category & Group 1 & Group 2 \\
\hline Focal & $15(16.4)$ & $7(16.2)$ \\
Multifocal & $11(12.0)$ & $5(11.6)$ \\
Diffuse & 0 & 0 \\
No lesions & $65(71.4)$ & $31(72.0)$ \\
\hline
\end{tabular}

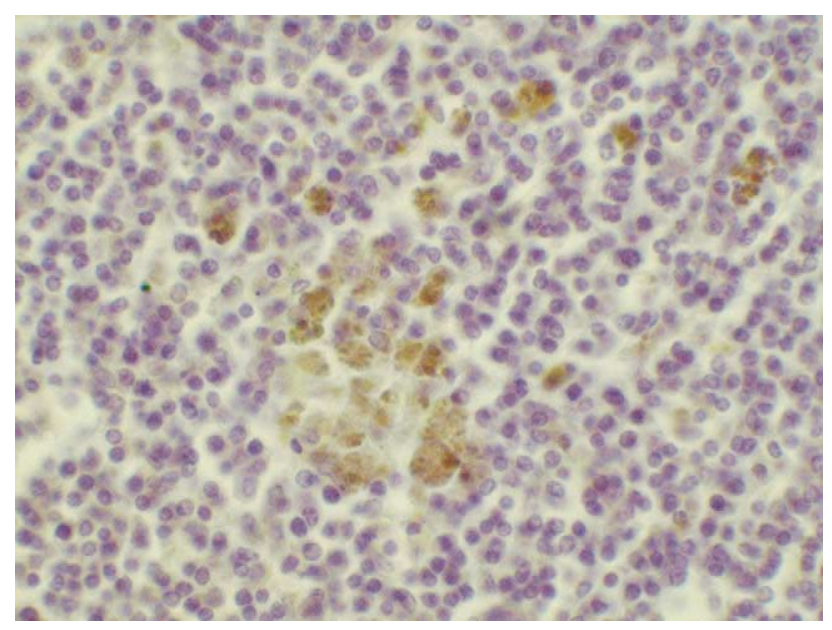

Fig. 1. Ileocecal lymph node, cattle, Immunoperoxidase staining in macrophages forming the granuloma $\times 400$. granulomas varied from 1 to 10 . From the animals with paratuberculosis lesions, Map was confirmed by immunohistochemistry in 24 from group 1 and 11 from group 2. Labelling was detected in macrophages within and around the granulomas (Fig. 1). The cells did not show intracytoplasmic staining with $\mathrm{ZN}$ and entire mycobacteria could also not be demonstrated by $\mathrm{ZN}$ or using immunohistochemical methods.

Although cross reactivity of the antibody with other mycobacteria cannot be excluded, the positive immunohistochemical reaction together with the location and histological features of the granulomatous lesions are considered as indicators highly consistent with Map infection. Intestinal infections with $M$. bovis or M. avium are considered rare in cattle and the morphology of the granulomatous lesions are characterised mainly by caseous necrosis (García Marín et al., 1995; Nieberle and Cohrs, 1966), features not observed in this work. Moreover, in a recent study, lesions similar to those described here, have been found to be positive for Map isolation by culture or identification by PCR (Pérez et al., 2002).

A variety of lesions associated with acquired sheep paratuberculosis has been described (Carrigan and Seaman, 1990; Pérez et al., 1996), mostly in subclinical cases and with specific lesions located exclusively in the intestinal lymphoid tissue and/or related lamina propria. Using the classification proposed for sheep, we found that $28.6 \%$ of cattle positive to the tuberculin skin test demonstrated focal paratuberculosis lesions with no clinical signs of the disease. A similar proportion $(27.9 \%)$ of lesions was, however, present in animals negative to the tuberculin test. Within the limits of the procedures employed, these findings suggest that the relationship of focal and multifocal lesions in cattle positive to the bovine tuberculin test is not significant. Focal lesions, similar to those encountered in this study, have been found in the initial phases of experimental paratuberculosis infections in sheep (Juste et al., 1994; Nisbet et al., 1962), and normally occur during the first months of life (Clarke, 1997), but in our work all the cattle were adults. Although the possibility of a recent infection cannot be excluded, it seems more likely that these focal lesions might represent persistent forms of Map infections taking place in the first weeks of life, as has been suggested in sheep (Pérez et al., 1996) and goats (Corpa et al., 2000). The focal lesions, in our work, did not contain complete acid-fast bacteria and, despite the existence of probably specific Map antigens within the macrophages, were not considered sufficient to induce tuberculosis cross-reactivity in the single skin test.

\section{Acknowledgements}

The authors would like to thank Dr. Lucas Dominguez from the Departamento de Microbiología, Facul- 
tad de Veterinaria, Madrid for kindly supplying the strain A-82. This work was supported by Ministerio de Educación y Ciencia. Grant No. 1FD97-0187.

\section{References}

Carrigan, M.J., Seaman, J.T., 1990. The pathology of Johne's disease in sheep. Australian Veterinary Journal 67, 47-50.

Chiodini, R.J., Van Kruiningen, H.J., Merkal, R.S., 1984. Ruminant paratuberculosis (Johne's disease): the current status and future prospects. Cornell Veterinarian 74, 218-262.

Clarke, C.J., 1997. The pathology and pathogenesis of paratuberculosis in ruminants and other species. Journal of Comparative Pathology 116, 217-261.

Corpa, J.M., Garrido, J., García Marin, J.F., Pérez, V., 2000. Classification of lesions observed in natural cases of paratuberculosis in goats. Journal of Comparative Pathology 122, 255-265.

García Marín, J.F., Chávez, G., Pérez, V., Badiola, J.J., 1995. Pathological findings in lambs experimentally infected with $\mathrm{Myco-}$ bacterium avium subsp avium, Mycobacterium avium subsp silvaticum and Mycobacterium subsp paratuberculosis. In: Chiodini, R.J., Collins, M.T., Bassey, E.O.E. (Eds.), Proceedings of the Fourth International Colloquium on Paratuberculosis. Evergreen Press, Rhode Island, USA, p. 208.

Juste, R.A., García Marin, J.F., Peris, B., Sáez de ocáriz, C., Badiola, J.J., 1994. Experimental infection of vaccinated and non-vaccinated lambs with Mycobacterium paratuberculosis. Journal of Comparative Pathology 110, 185-194.
Juste, R.J., Garrido, J.M., Aduriz, G., Moreno, B., Geijo, M.V., García-goti, M., Orgiza, J.A., 2000. Prevalencia de la paratuberculosis bovina en España. In: Proceedings of the Sixth International Congress of Bovine Medicine of ANEMBE, Santiago 2000, pp. 282-284.

Kohler, H., Gyra, H., Zimmer, K., Drager, K.G., Burkert, B., Lemser, B., Hausleithner, D., Cubler, K., Klawon, W., Hess, R.G., 2001. Immune reactions in cattle after immunization with a Mycobacterium paratuberculosis vaccine and implications for the diagnosis of $M$. paratuberculosis and $M$. bovis infections. Journal of Veterinary Medicine and Infection Disease Veterinary Public Health 48, 185-195.

Monaghan, M.L., Doherty, M.L., Collins, J.D., Kazda, J.F., Quinn, P.J., 1994. The tuberculin test. Veterinary Microbiology 40, 111124.

Nieberle, K., Cohrs, P., 1966. Tuberculosis. In: Textbook of the special pathological anatomy of domestic animals. Pergamon Press Ltd., London.

Nisbet, D.I., Gilmour, N.J.L., Brotherson, J.G., 1962. Quantitative studies of Mycobacterium johnei in tissues of sheep. III. Intestinal histopathology. Journal of Comparative Pathology 72, 80-91.

Pérez, V., García, J.M., Badiola, J.J., 1996. Description and classification of different types of lesion associated with natural paratuberculosis infection in sheep. Journal of Comparative Pathology $114,107-122$.

Pérez, V., González, J., Geijo, M.V., Reyes, L.E., Garrido, J.M., Corpa, J.M., García Pariente, C., García Marín, J.F., 2002. Pathological forms and peripheral immune responses in natural bovine paratuberculosis. In: Proceedings of the Seventh International Colloquium on Paratuberculosis. Bilbao (Spain), June 2002, p. 50. 